\title{
Growth Measurement of a Community of Strawberries Using Three-Dimensional Sensor
}

\author{
Satoshi Yamamoto, Shigehiko Hayashi and Shogo Tsubota \\ Bio-oriented Technology Research Advancement Institution, NARO \\ 1-40-2, Nisshin, Kita, Saitama 331-8537, Japan
}

(Received February 6, 2014; Accepted July 2 2014)

\begin{abstract}
The movable bench cultivation system is a method of increasing area productivity and minimizing the energy needed in a greenhouse. In the system, all plants pass through the same watering point every day. This point should thus be the ideal place to measure precise plant growth information. In this study, we construct an experimental system for a 3D measurement of a community of strawberries cultivated on a 1-meter-long bench. The measurement was made every two to seven days from October 23, 2013 to January 13, 2014. As a result, we obtained 31 images of 10 plant beds. The estimation error of maximum plant height ranged from $-30 \mathrm{~mm}$ to $32 \mathrm{~mm}$, and that of width ranged from $-42 \mathrm{~mm}$ to $40 \mathrm{~mm}$. To visualize the growth information effectively, a $2 \mathrm{D}$ histogram of the distribution of the $3 \mathrm{D}$ points of the plant community was also calculated.
\end{abstract}

Keywords : depth sensor, long-term observation, movable bench, 3D reconstruction

\section{INTRODUCTION}

Monitoring plant health is an important technique to guarantee high quality and quantity of agricultural production. In case of greenhouse-grown strawberry plants, farmers generally measure the plants' height, number of leaves, and area of a single leaf to obtain growth information and estimate the plant's health. These indices have been used successfully for many years in traditional strawberrygrowing. However, this method of monitoring plant health in an industrial-scale greenhouse would take up a lot of time. Furthermore, traditional growth information is not usually obtained every day. Even when the information is obtained, it involves taking only a sample value, which does not accurately report the condition of every plant.

A circulating-type movable bench system for strawberry cultivation has been studied in Japan (Yoshida et al., 2008; Hayashi et al., 2011; Saito et al., 2012). With this cultivation system, all plants pass daily through a single location, called the access point, to be watered. The access point is thus the ideal place for continually and precisely obtaining information on the growth of strawberry plants. Machine vision is a promising technique for effectively monitoring every plant at the access point, since it enables non-destructive measurement. For effective plant health monitoring, many imaging techniques such as digital color imaging, multi-spectral imaging, thermal imaging and fluorescence imaging have been investigated (Takayama and Nishina, 2009).

On the other hand, the availability of low-cost depth sensors that generate depth images is on the rise because of the development of natural user interfaces (NUIs), as seen in Microsoft's Kinect sensor, which is commonly used as a motion controller for TV games. The accuracy of the Kinect sensor has been reported as a percentage of measurement error: between $-2 \mathrm{~cm}$ to $2 \mathrm{~cm}$ is $90.9 \%, 82.9 \%$ and $81.2 \%$ for data along the $\mathrm{x}, \mathrm{y}$ and $\mathrm{z}$ axes of the camera coordinates, respectively, using an uncalibrated Kinect sensor (Khoshelham and Elberink, 2012). In the camera coordinates, $\mathrm{x}$ and $\mathrm{y}$ axes are included in the CMOS plane of the camera. Namely, $x$ axis is horizontal direction in the plane, and $y$ axis is vertical direction. Direction of $\mathrm{z}$ axis is equivalent to the normal vector of the plane. For the leaf segmentation in rosebushes and the measurement of the leaf angle of ornamental plants, the low-cost depth sensor has been applied (Chéné et al., 2012). The Kinect sensor of these researches was equipped with an active-stereo system to obtain depth information. In 2014, new Kinect sensor was released which measures the depth by way of timeof-flight method. It is easily predicted that more researches will be conducted using the new sensor in the foreseeable future.

We have developed a plant growth measurement method for strawberries using the active-stereo type Kinect sensor and have investigated the measurement accuracies of plant height and width and the area of leaves using a potted strawberry plant (Yamamoto et al., 2012). In this study, we propose an algorithm for a 3D measurement of a plant community of strawberries on a 1-meter long bench. We then report the results of a three-month observation of the plant community.

Corresponding author: Satoshi Yamamoto, fax: + 81-48-654-7137, e-mail : syamamot@affrc.go.jp 

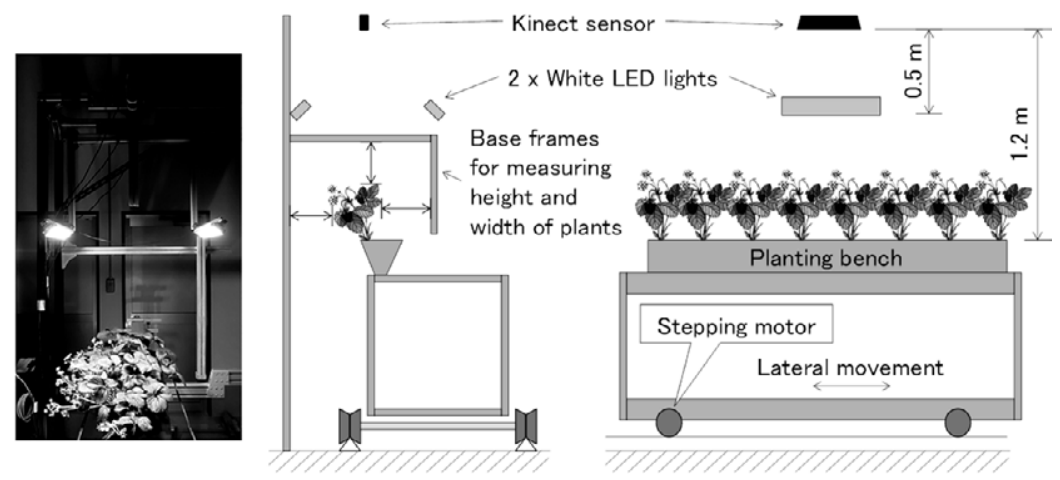

Fig. 1 Experimental system for 3D measurement of plant community of strawberries.
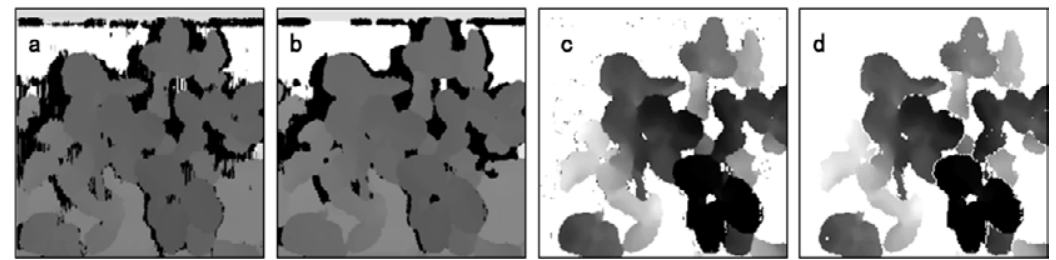

Fig. 2 Noise reduction of tiled depth image. (a) Tiled raw data. (b) Tiled median data. (c) Extracted leaves including small discontinuous regions. (d) A result of noise reduction.

\section{MATERIALS AND METHODS}

The experimental system consists of a Kinect sensor as a depth sensor, a light source and a wheeled platform driven along rails by a stepping motor (Fig. 1). While the system is capturing images, the velocity of the wheeled platform is set to approximately $16 \mathrm{~mm} \mathrm{~m}^{-1}$. The start and stop position of the wheeled platform is recorded. For a manual measurement of the height and the width of the strawberry plants, base frames are set on the system. For a movable bench system in a greenhouse where the benches are longer than 3 meters, the depth sensor will not be able to capture the entire bench in a single shot. A line image of 480 pixels is therefore cropped from the $640 \times 480$-pixel image generated at a rate of $30 \mathrm{fps}$ from the depth sensor. These line images are then tiled as for a line camera while the wheeled platform carries plants past at constant speed. A tiled color image is also obtained, since the Kinect sensor captures depth and color images simultaneously. Although a bench longer than a meter can be captured by tiling, a great deal of noise was observed in the tiled depth image, especially around the boundaries of objects, as shown in Fig. 2. To reduce noise, 11 line images were cropped from 11 VGA images by shifting one pixel, and a median image of these line images was created and tiled. After the leaves were extracted from the tiled median image, there were still numerous small discontinuous regions with an area of fewer than 10 pixels. These regions were labeled and eliminated from the target region.

Although the depth sensor constantly generates images at $30 \mathrm{fps}$, the tiling speed of line images varies slightly among the tiled images. The calibration image shown in Fig. 3 and the position data of the stepping motor was therefore used to correct the differences. Finally, the 3D positions of the extracted points were calculated using the

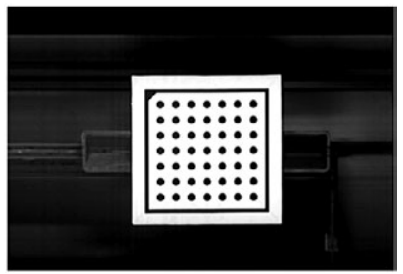

Fig. 3 Tiled color image of a calibration plate of $0.5 \mathrm{~m}$.

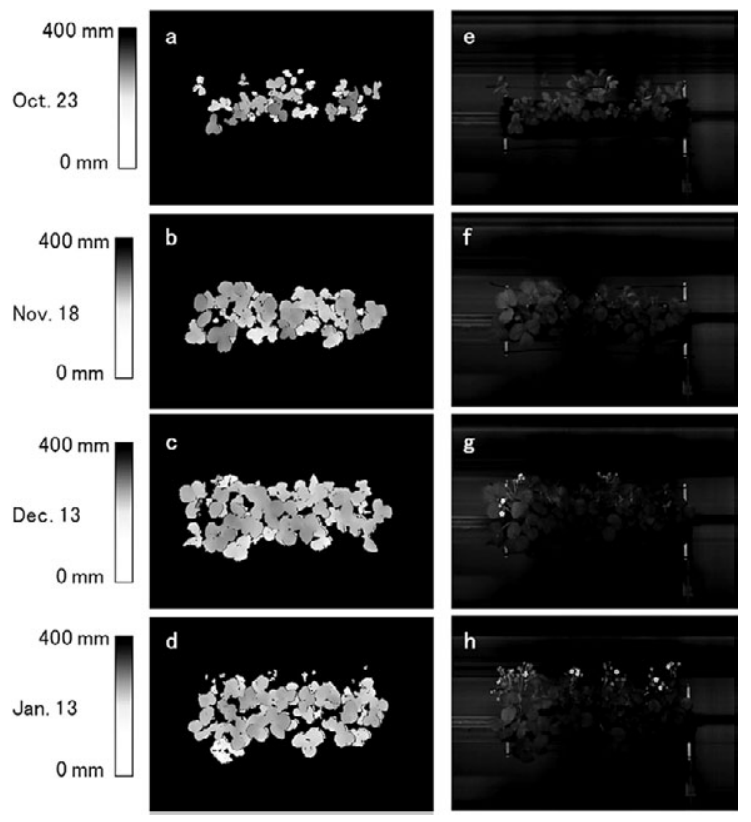

Fig. 4 Images of long-term observation. (a)-(d) extracted leaf images from tiled depth images. The vertical axis of the extracted leaf images is the height of plants from the bed surface. (e)-(h) tiled color images.

depth data, the calibration parameters of the depth sensor and the distance traveled by the wheeled platform.

To measure the growth information of the strawberry 

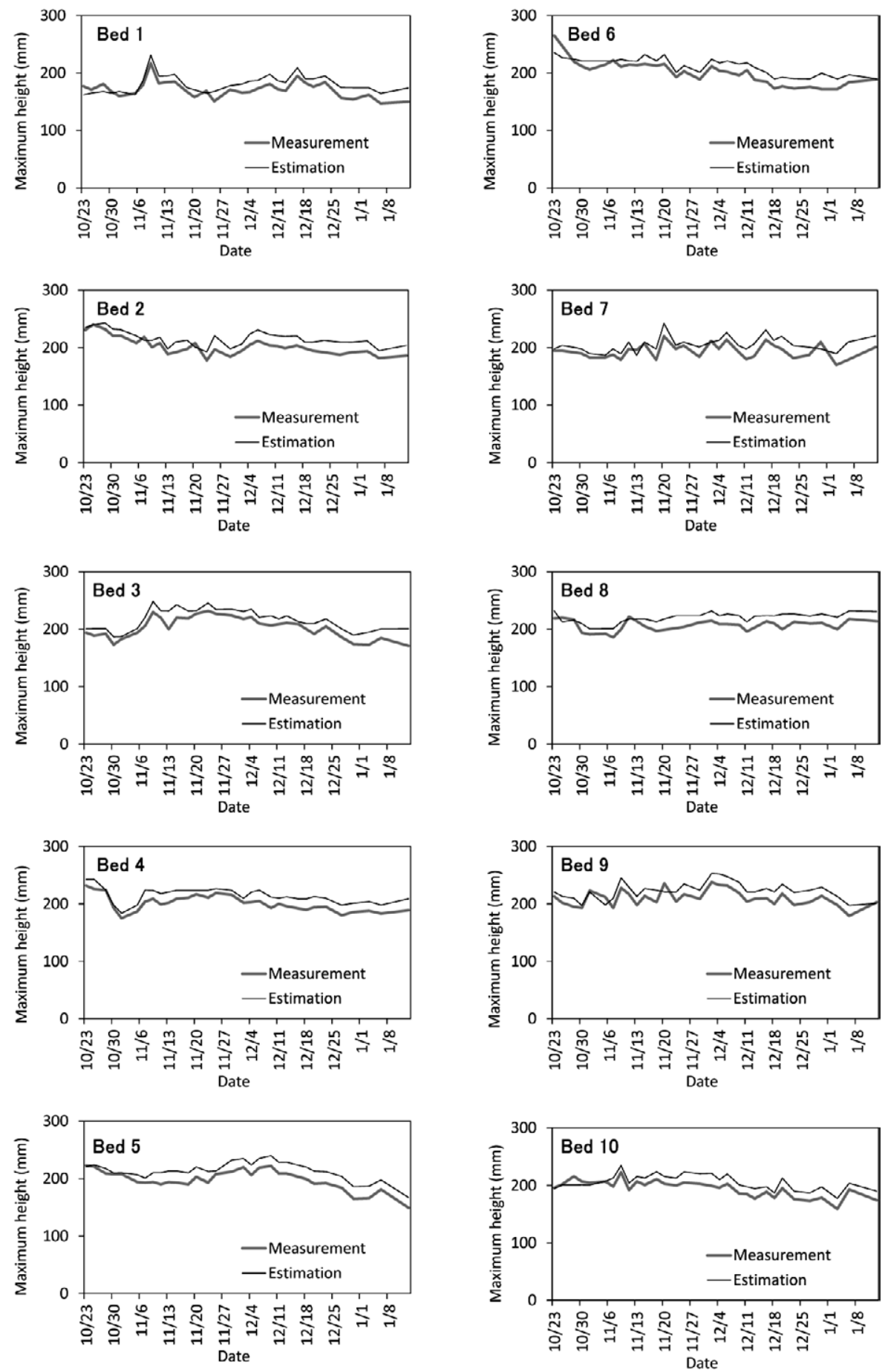

Fig. 5 Changes of maximum height of plant communities grown on a 1 meter-long bed from Oct. 23, 2013 to Jan. $13,2014$.

plants, we had 10 beds, each a meter long, on which eight strawberry plants of the cv. Beni-hoppe had been grown. They were planted on October 16, 2013, and were measured every two to seven days from October 23, 2013 to January 13, 2014. As a result, we obtained 31 images of each bed. To evaluate the accuracy of the experimental system, the maximum plant height and width at each bench was measured manually.

\section{RESULTS AND DISCUSSIONS}

Figure 4 shows an example of extracted leaf images from the tiled depth images and the tiled color images obtained by the long-term observation.

Comparing these leaf images, it seemed that the growth of the plant community could be understood in an intuitive way. When the flowers bloomed in January 2014, however, some of them were recognized as leaves by the algorithm. In the colored images, the precise measurement of color information of the leaves was difficult because the exposure setting of the Kinect sensor changed automatically during the scanning operation.

The maximum plant height and width of each bed are plotted with time in Figs. 5 and 6. 

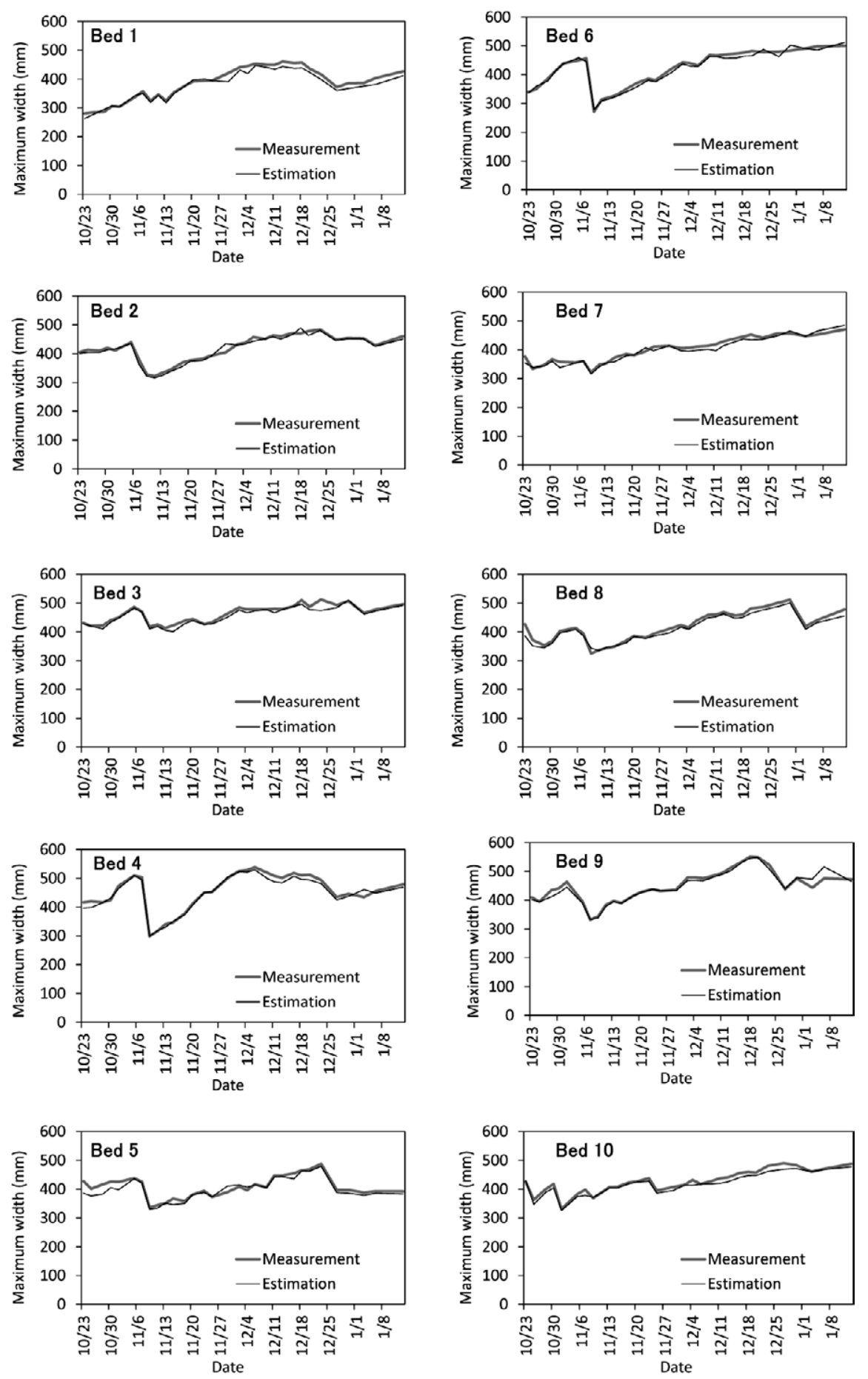

Fig. 6 Changes of maximum width of plant communities grown on a 1 meter-long bed from Oct. 23, 2013 to Jan. $13,2014$.

The estimation error of plant height ranged from -30 $\mathrm{mm}$ to $32 \mathrm{~mm}$, and that of width ranged from $-42 \mathrm{~mm}$ to $40 \mathrm{~mm}$. The average and the standard deviation of the estimation error of plant height were $12.4 \mathrm{~mm}$ and $8.4 \mathrm{~mm}$ respectively, and those for plant width were $-6.7 \mathrm{~mm}$ and $9.8 \mathrm{~mm}$ respectively. We observed that the leaf tips could not be detected when the leaf was vertically inclined. These errors seemed to be small enough compared with the changes of growth information of the community during the long-term observation, and thus plant health could be diagnosed using estimated plant height and width.

To monitor the three-dimensional features of each plant in the community, the visualization of the distribution of the 3D points of each plant seemed effective. To divide the plant community on the bed into eight parts, we simply separated the bed into eight blocks, each $0.125 \mathrm{~m}$ long, using the position data of the 3D points. Figure 7 shows the two-dimensional histograms of plant No. 1 and No. 4 from bed No. 6 during the long-term observation. The graphical data seemed to intuitively indicate the height and the width. We could also observe the activity of the plants using the distribution of the $3 \mathrm{D}$ points, which may correlate to the volume or the surface area of the plant. If the range of distribution of leaves on the histogram is ideal, the 


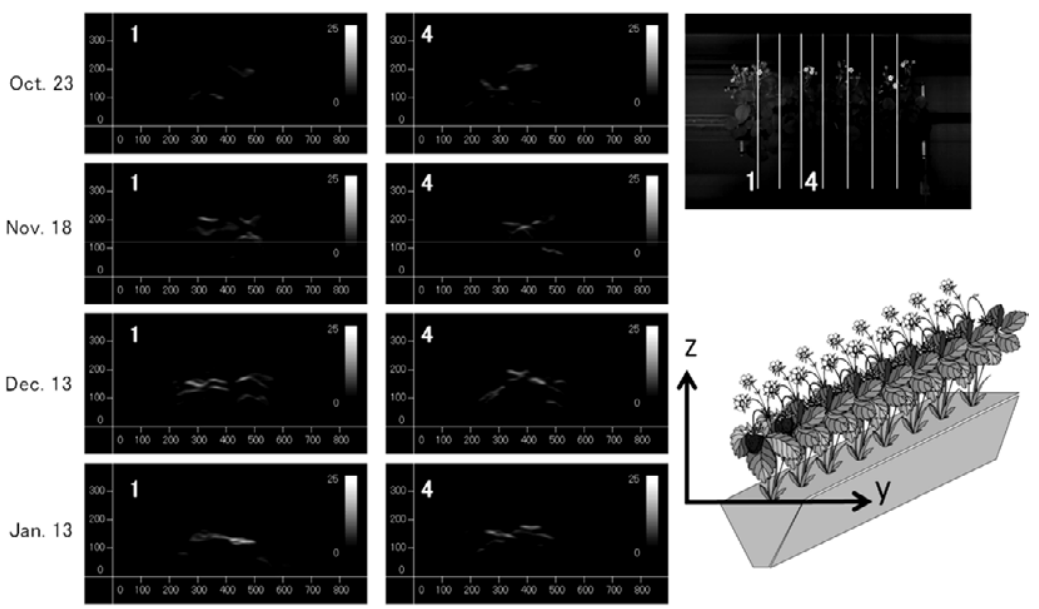

Fig. 7 Comparison of 2D histogram of plant No. 1 and No. 4. of bed No. 6 from Oct. 23, 2013 to Jan. 13, 2014. In the histograms, scale of horizontal axis is y coordinate in $\mathrm{mm}$, and that of vertical axis is $\mathrm{z}$ coordinate in $\mathrm{mm}$. The gradation scale shows the frequency of $3 \mathrm{D}$ points.

diagnosis of the strawberry plants will be possible by evaluating how close the $3 \mathrm{D}$ points come to the optimal range.

\section{CONCLUSION}

For precise growth measurement of the strawberry plants at the access point of the movable bench system, a basic study was conducted into the feasibility of 3D measurement of plant community grown on a 1-meter-long bed. It was initially confirmed that a $3 \mathrm{D}$ point cloud of the plant community could be obtained using our experimental system, which consists of a depth sensor, a light source and a wheeled platform. As a result of long-term observation, growth information was visualized by the leaf images extracted from tiled depth images. It was revealed that the estimation error of maximum height and width were small enough compared to the change of growth information during the long-term observation, and the growth was evaluated using a two-dimensional histogram of the distribution of $3 \mathrm{D}$ points. Further experiments are currently being conducted to reduce the considerable workload imposed by traditional monitoring and achieve optimal management of strawberry plants.

\section{ACKNOWLEDGEMENTS}

This research was carried out through KAKENHI
(24580382), funded by the Japanese Society for the Promotion of Science.

\section{REFERENCES}

Chéné, Y., Rousseeau, D., Lucidarme, P., Berrtheloot, J., Caffier, V., Morel, P., Belin, É., Chapeau-Blondeau, F. 2012. On the use of depth camera for 3D phenotyping of entire plants. Comput. Electron. Agric. 82: 122-127.

Hayashi, S., Saito, S., Iwasaki, Y., Yamamoto, S., Nagoya, T., Kano, K. 2011. Development of circulating-type movable bench system for strawberry cultivation. Jpn. Agric. Res. Q. 45: $285-293$.

Khoshelham, K., Elberink, S. O. 2012. Accuracy and resolution of Kinect depth data for indoor mapping applications. Sensors 12: 1437-1454.

Saito, S., Hayashi, S., Yamamoto, S., Iwasaki, Y., Takahashi, N. 2012. Study on work efficiency and practical scale of a movable bench system for strawberry cultivation. J. Jpn. Soc. Agric. Machinery 74: 457-464.

Takayama, K., Nishina, H. 2009. Chlorophyll fluorescence imaging of the chlorophyll fluorescence phenomenon for plant health monitoring. Environ. Control Biol. 47: 101-109.

Yamamoto, S., Hayashi, S., Saito, S., Ochiai, Y. 2012. Measurement of growth information of a strawberry plant using a natural interaction device. ASAE 12: 1341108.

Yoshida, H., Yamamoto, S., Hayashi, S., Iwasaki, Y., Urushiyama, Y. 2008. Development of a movable bench system for high-density cultivation of strawberries. J. Jpn. Soc. Agric. Machinery 70: 98-106. 